\title{
Natalizumab in the treatment of Crohn's disease
}

\author{
Danila Guagnozzi \\ Renzo Caprilli \\ Gastroenterology Unit, Department \\ of Clinical Sciences, University \\ of Rome "La Sapienza", Rome, Italy
}

\begin{abstract}
The pathogenesis of Crohn's disease (CD) is multifactorial and the activation of specific pathways of immunological system is important. In particular, the adhesion molecules (integrins) mediate the selective binding between the leukocytes and the endothelial cells regulating the migration of leukocytes into the normal and inflamed intestine. Selective adhesion molecule inhibitors interfere with the migration of leukocytes to the sites of inflammation by targeting adhesion molecules ( $\alpha 4$-integrin or $\alpha 4 \beta 7$-integrin). Natalizumab is a humanized IgG4 anti- $\alpha 4$-integrin monoclonal antibody that inhibits both $\alpha 4 \beta 7$-integrin/mucosal addressin-cell adhesion molecule-1 (MadCAM-1) interaction and $\alpha 4 \beta 1 /$ vascular-cell adhesion molecule- 1 (VCAM-1) binding. Pooled data from the four studies, analyzed in a Cochrane review, suggest that natalizumab is effective for induction of clinical response and remission in patients with moderately to severely active CD. In particular, natalizumab may be beneficial for patients with active inflammation or chronically active disease despite the use of conventional therapies with high level of C-reactive protein values at baseline time. Nevertheless, many problems about the utilization of natalizumab in CD remain unsolved (such as the high placebo response, the final definition of dosage and timing schedule, the definition of outcomes and the development of adverse events).
\end{abstract}

Keywords: Crohn's disease, biological therapy, natalizumab, progressive multifocal leukoencephalopathy

\section{Introduction}

The etiology of inflammatory bowel diseases (IBD) has not yet been fully addressed, but there has been remarkable progress in the understanding of this field in the past decade. In normal bowel, the immune reaction is sophistically regulated while keeping a balance between the effectors and the regulators, and as a result the homoeostasis of the gut is maintained. A lot of evidence indicates that mucosal immunity is dysregulated in the bowel of IBD patients.

In particular, the pathogenesis of Crohn's disease (CD) is multifactorial, including genetic factors, environmental factors, and immunological factors. For the development of intestinal inflammation, the activation of the specific pathways of immunological system are important and the leukocyte trafficking to the gut is an important step (Fiocchi 1998). Adhesion molecules, such as integrins, mediate the selective binding between the leukocytes and the endothelial cells and thus the migration of leuokocytes into normal and inflamed intestine. The main classical medical treatments for IBD are steroids and immunosuppressive agents which non-specifically reduce immunity and inflammation. Recent advances in the understanding of the mechanisms of bowel inflammation have led to a recent trend in development of biological therapies which selectively inhibit the action of molecules essential to the inflammatory process (Nakamura et al 2006) (Figure 1). Major targets for such therapies are inflammatory cytokines and their receptors, and adhesion molecules. Selective adhesion molecule inhibitors interfere with the migration of leucocytes to the sites of inflammation by targeting adhesion molecules, such as $\alpha 4$-integrin or $\alpha 4 \beta 7$-integrin. In particular, 


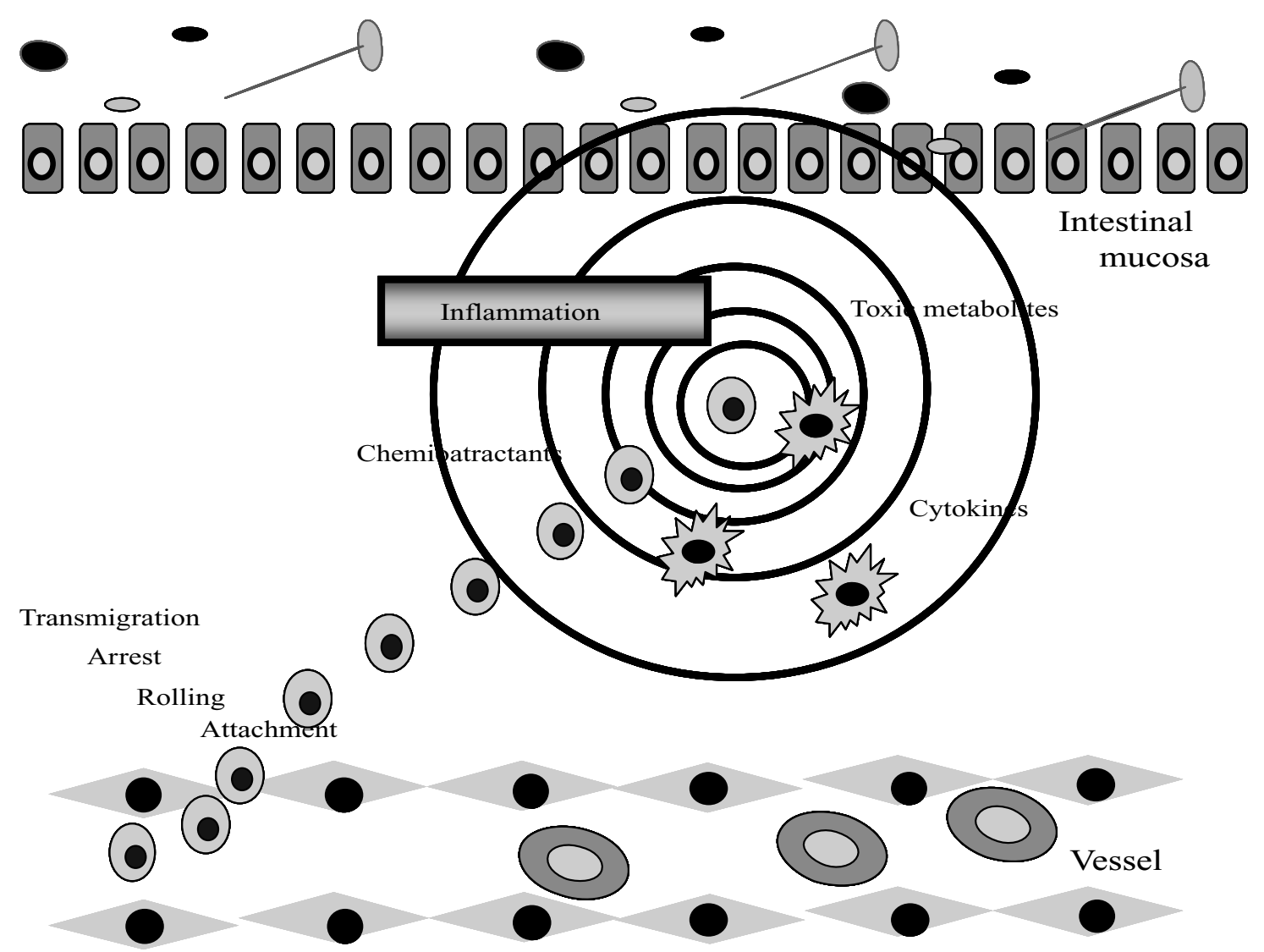

Figure I Adhesion molecules (integrins) mediate the selective binding between the leukocytes and the endothelial cells to migrate from the vessels to the inflamed intestinal muocsa, through four phases: attachment, rolling, arrest, and transmigration. The toxic metabolites, cytokines, and chemioactractans, produced by the inflammation source, induce and drive the trafficking of immune cells to final destination.

natalizumab, a humanized IgG4 anti $\alpha 4$-integrin monoclonal antibody containing approximately $5 \%$ mouse-derived protein, inhibits both $\alpha 4 \beta 7$-integrin/MAdCAM-1 interaction and $\alpha 4 \beta 1$ /VCAM-1 binding (Figure 2). The US Food and Drug Administration has approved natalizumab for multiple sclerosis with the requirement of mandatory participation in a risk-management and registry program.

\section{Therapeutic targets of natalizumab}

The integrins are a family of cell-surface glycoproteins involved in the adhesion, migration, and activation of immune cells. In particular, the integrins are adhesion molecules that confer mechanical stability on interactions between cells and their environment (Hynes 2002). They also act as cellular sensors and signaling molecules. All integrins are composed of non-covalently linked $\alpha$ and $\beta$ chains. The $\alpha 4-$ integrin is expressed at a moderate or high level on almost all lymphocytes and to a lesser extent on monocytes and eosinophils. The $\alpha 4$-integrin chain dimerizes with either the $\beta 1$ chain or the $\beta 7$ chain. The $\alpha 4 \beta 1$-integrin is also known as very late antigen 4 or cCD $49 \mathrm{~d}-\mathrm{CD} 29$, and the $\alpha 4 \beta 7$ - integrin is sometimes referred to as lamina propria-associated molecule-1. Natalizumab binds the $\alpha 4$ chain irrespective of its associated $\beta$ chain and probably has therapeutic effects because it blocks the ability of $\alpha 4 \beta 1$ and $\alpha 4 \beta 7$ to bind to their respective endothelial counter-receptors, vascular-cell adhesion molecule-1 (VCAM-1), and mucosal addressincell adhesion molecule-1 (MAdCAM-1). These molecular interactions are required for lymphocytes to enter the central nervous system (mediated by $\alpha 4 \beta 1$ and VCAM- 1 ) and the intestine (mediated by $\alpha 4 \beta 7$ and MAdCAM-1). In fact, human and animal studies have suggested that the interaction between $\alpha 4 \beta 7$ and MAdCAM- 1 is particularly important in mediating leukocyte homing to gut mucosa. Furthermore, studies of patients with IBD show that endothelial cells extracted from inflamed intestinal mucosa demonstrate increased $\alpha 4$ dependent adhesiveness to leukocytes from patients with IBD in vitro (Binion et al 1998).

It is now well established that the recruitment of leukocytes from the blood into virtually every tissue is regulated by sequential engagement of adhesion and signaling molecules on leukocytes and endothelial cells (Springer 1994; 


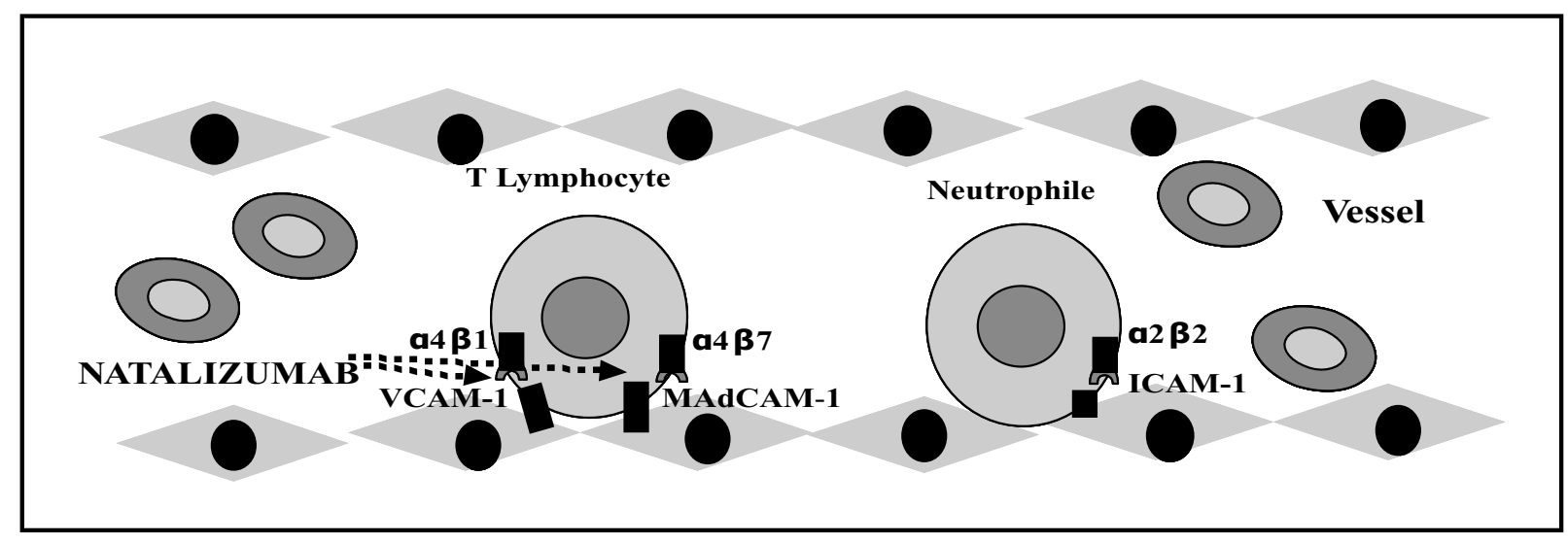

Figure 2 Pharmacological targets of natalizumab. Several adhesion molecules are espressed on the surface of endothelial and immune system cells. In particluar, natalizumab inhibits the interaction between $\alpha 4 \beta$ I and $\alpha 4 \beta 7$ integrins expressed on the surface of T lymphocyte cells, andVCAM-I and MAdCAM-I expressed on the surface of endothelial cells.

Butcher and Picker 1996; Von Andrian and Mackay 2000), described above. Leukocytes make an initial adhesive contact that allows them to slow down and roll along the vascular wall. This step, which is readily reversible, can be mediated by selectins, $\alpha 4$ integrins, or both. In order for the rolling leukocyte to stop, it must receive an activating signal, such as a signal from a chemokine, which switches integrins to a high affinity state and allows the cell to arrest itself (Springer 1994; Butcher and Picker 1996; Von Andrian and Mackay 2000). Leukocytes are recruited into a tissue only if they are successful at undergoing each step. The two $\alpha 4$ integrins and their endothelial counter-receptors have a unique role in this multi step cascade because they are the only molecules known to mediate both rolling (when the integrins are in a low-affinity state) and arrest (when they are in a high-affinity state) (Alon et al 1995; Berlin et al 1995) (Figure 1).

Two main indications are currently investigated for natalizumab, multiple sclerosis and CD.

\section{Multiple sclerosis (human equivalent of animal autoimmune encephalomyelitis)}

The earliest evidence of $\alpha 4$-integrin mediated central nervous system disease came from studies of autoimmune encephalomyelitis, a T-cell mediated autoimmune disease that resembles multiple sclerosis, in animals (Yednock et al 1992). Antibodies to $\alpha 4$ integrins reduce cellular infiltration, inhibit the development of autoimmune encephalomyelitis, and halt the progression of disease or even reverse existing symptoms by preventing inflammatory cells from crossing the blood - brain barrier. These findings suggest that $\alpha 4$-integrin inhibitors should block the egress of pathogenic T-cells into unaffected regions of the central nervous system, where their stimulation by antigen would precipitate the inflammation cascade. Such blockade may explain the effectiveness of natalizumab in preventing the formation of new lesions in patients with multiple sclerosis (Miller et al 2003), which is the human equivalent of auto-immune encephalomyelitis.

\section{Crohn's disease (CD)}

The effects of natalizumab in patients with $\mathrm{CD}$ are likely to involve inhibition of the binding of $\alpha 4 \beta 7$ to MAdCAM-1. MAdCAM-1 is selectively expressed in venules in the gut and gut-associated lymphoid tissue (Butcher and Picker 1996) (Figure 2). Animal models of colitis have shown that antibodies to $\alpha 4 \beta 7$ or MAdCAM-1 attenuate T-cell-mediated intestinal inflammation (Hesterberg et al 1996; Picarella et al 1997). However, VCAM-1 is also induced in inflammatory bowel disease and predominates in some animal models of experimental colitis (Soriano et al 2000). The extent to which natalizumab exerts its therapeutic effects by inhibiting $\alpha 4 \beta 1$ in addition to its blockade of $\alpha 4 \beta 7$ remains to be determined. Whatever the precise mechanism of natalizumab's efficacy in patients with $\mathrm{CD}$, the drug had only partial anti-inflammatory effects during the treatment periods (Ghosh et al 2003), suggesting that intestinal inflammation involves other pathways of leukocytes recruitment. Indeed, the inflammatory infiltrate in $\mathrm{CD}$ is dominated by neutrophils, which do not normally express $\alpha 4$ integrins, but instead rely on $\beta 2$ integrins to travel to sites of inflammation (Springer 1994). Neutrophils can express $\alpha 4 \beta 1$ once they have emigrated out of blood vessels (Kubes et al 1995), but it is questionable whether the inhibition of $\alpha 4$ integrins at this stage can interfere with leukocytes-mediated disease. The T-cells are essential for inducing the secondary signaling molecules in the gut, such as cytokines and chemokines that are needed to sustain neutrophil recruitment (Hesterberg et al 1996). 


\section{Clinical studies in Crohn's disease}

Preclinical studies have shown that monoclonal antibodies against $\alpha 4$-integrin reduce inflammation and symptoms of disease in tamarins with IBD (Podolsky et al 1993; Hesterberg et al 1996). After that, 2 pilot studies showed an improvement in signs and symptoms of patients with CD or ulcerative colitis (UC) treated with natalizumab (Gordon et al 2001, 2002). Four randomized, double-bind, placebo-controlled trials were then published and will be analyzed in detail in this review.

\section{Study of Gordon et al (200I)}

In this study, 35 adults with a confirmed diagnosis of $\mathrm{CD}$ were screened and 30 patients randomized to receive the treatment from 2 centers in the UK. All patients included presented a clinical evidence of mild to moderately active $\mathrm{CD}$ (defined by a Crohn's Disease Activity Index [CDAI] of $>151$ and $<450$ ). Oral corticosteroids were allowed if the dose was stable within 2 weeks of study entry. Patients receiving 5-ASA drugs or azatioprine or 6-mercaptopurine were included if treatment had not been started within 2 months or the dose increased within 4 months of study entry. Exclusion criteria included: pregnancy or breast-feeding; patients weighing more than $100 \mathrm{~kg}$; patients with CDAI score $>450$; patients treated with cyclosporine, methotrexate, or tracolimus therapy; patients who had abdominal surgery within 3 months of entry or were considered likely to require surgery within 3 months of study entry; patients with an ileostomy or colostomy; patients with laboratory-confirmed intestinal infection; patients with a current or past malignant neoplasm. Two groups of patients were randomized to receive: an intravenous infusion of natalizumab $(3 \mathrm{mg} / \mathrm{kg} ; \mathrm{n}=18)$ or placebo $(\mathrm{n}=12)$. The demographic characteristics of the patients at study entry were: $9 / 30(30 \%)$ with perianal disease; $9 / 30(30 \%)$ with ileal and colonic location; $8 / 30(27.7 \%)$ with colonic alone location; and 12/30 (40\%) with ileal alone location. The primary outcome was the change in mean CDAI at 2 weeks post infusion and clinical remission (defined as a CDAI score of less than 150). Other secondary outcomes included Inflammatory Bowel Disease Questionnaire (IBDQ), serum levels of C-reactive protein (CRP), erythrocyte sedimentation rate (ESR), full blood count, peripheral blood T-cells and B cells, serum natalizumab and anti-natalizumab antibody concentration. Patients were evaluated at study entry and at weeks $1,2,4,8$, and 12 post infusion. Three patients withdrew form the study before completing 12 weeks ( 2 for intervention ineffective and 1 for loss to follow-up). Natalizumab-treated patients achieved significant reductions in CDAI at $2(p=0.02)$ and $4(p=0.01)$ weeks after treatment compared with baseline, although results of between-groups comparisons with placebo-treated patients were not statistically significant. By 2 weeks $39 \%$ had experienced remission compared with $8 \%$ of the placebo group ( $\mathrm{p}=\mathrm{NS}$ ). Remission was sustained to at least week 12 in two of the natalizumab-treated patients. The two observed differences between groups were not statistically significant. A significant improvement in mean IBDQ score occurred from 121 points at baseline to 140 points at 4 weeks in patients who received natalizumab $(p=0.004)$. Patients who received natalizumab experienced a significant reduction in CRP at 2 and 4 weeks compared with baseline levels ( $\mathrm{p}=0.02$ at both time points) and a significant reduction in ESR at 4 weeks only $(p=0.04)$. These changes did not differ significantly from those in the placebo group. Two patients (11\%) developed transient low-level non-antiidiotypic antibodies to natalizumab, detectable only at a single visit during the 12-week trial follow-up period. The most common adverse events reported in at least $20 \%$ of patients during the 12 -week follow-up period were headache $(50 \%$ of each treatment group), CD exacerbation (39\% in natalizumab group and $42 \%$ in placebo group), and abdominal pain ( $22 \%$ in natalizumab group and $17 \%$ in placebo group). The frequency of these events did not differ significantly between groups. Two natalizumab patients and 1 placebo patient required surgical resection of $C D$ at 66,69 , and 70 days, respectively. A significant increase in mean circulating lymphocyte counts occurred at 1,2, and 4 weeks post infusion in natalizumab patients only. In this study the effects of natalizumab at a dose of $3 \mathrm{mg} / \mathrm{kg}$ appear to be relatively short-lived in patients with active $\mathrm{CD}$. This finding may be related to the finding that the half-life of natalizumab is 4.8 days in IBD patients, shorter than the 8.7 days observed in healthy volunteers (Elan Pharma 1997). In vitro leukocyte-saturation studies now suggest that a minimum serum concentration of approximately $3 \mu \mathrm{g} / \mathrm{mL}$ of natalizumab is required to produce appropriate saturation of at least $80 \%$ of membrane-bound $\alpha 4$ integrins (Elan Pharma Ltd, unpublished data, June 1999). The mean serum concentrations of natalizumab at 2 and 4 weeks post infusion were 4.91 and $0.99 \mu / \mathrm{mL}$, respectively, suggesting perhaps that blockade of $\alpha 4$ integrins was suboptimal after a single 3 $\mathrm{mg} / \mathrm{kg}$ dose and that larger and/or more frequent doses might result in improved efficacy.

\section{Study of Ghosh et al (2003)}

In this study, 248 adults ( $>18$ years of age) were recruited from 35 centers (Belgium, Czech Republic, Denmark, Germany, Israel, the Netherlands, Sweden, and the UK) for a 12-week induction trial. All patients included in the study 
had clinical evidence of moderate to severe $\mathrm{CD}$, defined by a CDAI of at least 220 but no more than 450. The exclusion criteria were: patients who received methotrexate, cyclosporine, or any investigational drugs within 3 months prior to randomization; patients taking stable doses of azathioprine or 6-mercaptopurine for less than 4 months prior to randomization; prior treatment with any antibody agent; current use of more than $25 \mathrm{mg}$ /day of oral prednisolone or another corticosteroid at an equivalent dose; elemental diet or parenteral nutrition; infectious or neoplastic disease of the bowel; bowel surgery within 3 months prior to randomization; the presence of an ileostomy; the presence of symptoms due mainly to fibrotic strictures; and a clinical impression that the patient would likely require abdominal surgery. The four treatment groups received 2 intravenous infusions 4 weeks apart at week zero and week 4 , with following schedule: 2 infusions of placebo $(n=63)$; 1 infusion of natalizumab (3 mg/kg), and 1 infusion of placebo $(\mathrm{n}=68)$; 2 infusions of natalizumab $(3 \mathrm{mg} / \mathrm{kg} ; \mathrm{n}=66)$ and 2 infusions of natalizumab $(6 \mathrm{mg} / \mathrm{kg} ; \mathrm{n}=51)$. The primary outcome variable was the proportion of patients who entered remission at week 6 (defining as a CDAI score of less than 150). The secondary outcome variable was the proportion of patients presenting clinical response (defining as a decrease in the CDAI score of at least 70 points from baseline). Other secondary outcome variables included the serum concentration of CRP, absolute neutrophil counts, serum antibodies against natalizumab, and health-related quality of life as measured by the IBDQ. The demographic characteristics of the patients at baseline were: $15.3 \%$ (38/248) fistulizing disease; 54.8\% (136/248) ileum and colon location; 23.8\% (59/248) colon disease; $21.4 \%$ (53/248) only ileum disease. Patients were evaluated at baseline and weeks 2, 4, 6, 8, and 12. Of the 244 patients who received at least 1 dose of the assigned study drug, 27 withdrew from the study before completing 12 weeks (ineligibility [2/27], adverse events [8/27], lack efficacy [7/27], patient's request [3/27], loss to follow-up [2/27], and investigator's decision [5/27]). The total numbers of patients who had adverse events during or after treatment were similar in the four groups ( $81 \%$ vs $77 \%$ vs $88 \%$ vs $78 \%$ ) with very high value, even if without any serious adverse events. Antibodies binding to natalizumab were detected in 13 natalizumab-treated patients (7\%) at week 12 .

The rate of remission in the group given 2 infusions of $6 \mathrm{mg} / \mathrm{kg}$ of natalizumab was not significantly different from the rate in the placebo group at week 6 (primary outcome) such as in the group given 1 infusion of natalizumab $(3 \mathrm{mg} / \mathrm{kg})$. At weeks 4 and 8 the remission rate among the patients who received two $6 \mathrm{mg}$ doses was significantly superior to the rate in the placebo group ( $29 \%$ vs $14 \%, p=0.028$ and $43 \%$ vs $16 \%, \mathrm{p}<0.001$, respectively). The group given 2 infusions of $3 \mathrm{mg} / \mathrm{kg}$ of natalizumab had a significantly higher rate of clinical remission at weeks $4,6,8$, and 12 than did the placebo group (29\% vs 14\% with $\mathrm{p}=0.027$, 44\% vs $27 \%$ with $\mathrm{p}=0.03,41 \%$ vs $16 \%$ with $\mathrm{p} \leqslant 0.001$, and $42 \%$ vs $27 \%$ with $\mathrm{p}=0.042$ ). All three natalizumab groups had a significantly higher rate of clinical response than did the placebo group at weeks 4, 6, 8 (with the higher value in the group received 2 infusions of $3 \mathrm{mg} / \mathrm{kg}$ with maximum response rate level of $71 \%$ at week 6 , with $\mathrm{p}<0.001$ ), and at week 12 for the two groups that received 2 infusions of natalizumab (61\% vs $43 \%, \mathrm{p}=0.033$ and $65 \%$ vs $43 \%, \mathrm{p}=0.018$ ). Two infusions of natalizumab administered 4 weeks apart resulted in more durable clinical response than a single infusion and 2 infusions of $6 \mathrm{mg} / \mathrm{kg}$ had no advantage over 2 infusions of $3 \mathrm{mg} / \mathrm{kg}$. All three natalizumab groups ( 1 infusion and 2 infusions at $3 \mathrm{mg} / \mathrm{kg}$ or $6 \mathrm{mg} / \mathrm{kg}$ ) had significant improvement in mean IBDQ scores at week 6, as compared to placebo (155 vs 145, $\mathrm{p}=0.008$ for 1 infusion of $3 \mathrm{mg} / \mathrm{kg}, 163$ vs $145, \mathrm{p}<0.001$ for 2 infusions of $3 \mathrm{mg} / \mathrm{kg}$, and 155 vs 145, p $<0.001$ for 2 infusions of $6 \mathrm{mg} / \mathrm{kg}$ ). By week 12, only the groups that received 2 infusions of natalizumab continued to have scores that were significantly higher than in placebo group (161 vs $145, \mathrm{p}=0.033$ and 155 vs $145, \mathrm{p}=0.018$ for 2 infusions of $3 \mathrm{mg} / \mathrm{kg}$ and 2 infusions of $6 \mathrm{mg} / \mathrm{kg}$, respectively). Patients treated with 2 infusions of natalizumab had a significant decline from baseline in the serum levels of CRP only at week 6 , as compared with the placebo group $(\mathrm{p}<0.05)$. Finally there was a sustained increase in the mean lymphocyte count, ranging from 1.3 to 1.9 times the baseline value in each natalizumab group, remaining within normal range. The increase in circulating lymphocytes in natalizumab-treated patients may be a manifestation of the interruption of $\alpha 4$ integrin-mediated migration of lymphocytes to extravascular lymphocytes. We can speculate that it should be a clinically significant mechanism of action of natalizumab.

\section{Study of Sandborn et al (2005)}

In the ENACT-1 study, 905 adult patients with moderate to severe CD (defined by a baseline CDAI score of 220 to 450 points) were recruited from 142 centers in North America, Europe, and selected countries around the world for a 12week induction trial. Concomitant medication, including stable doses of 5-ASA drugs, prednisone $(25 \mathrm{mg}$ /day), budesonide, azathioprine, 6-mercaptopurine, methotrexate, and antibiotics were permitted. Exclusion criteria included: 
patients with short bowel syndrome, an ileostomy, a total colectomy, a stricture with obstructive symptoms, draining fistulas, abdominal abscess, previous natalizumab treatment, or previous anti-tumor necrosis factor $\alpha$ (TNF $\alpha)$ therapy within 3 months prior to study entry. Enrolment of patients who were non-responders to anti-TNF $\alpha$ treatment was limited to a maximum of $30 \%$ of total study enrolment. Patients were randomized to receive an infusion of either $300 \mathrm{mg}$ of natalizumab $(n=724)$ or placebo $(n=181)$ for a total of 3 infusions given at weeks 0,4 , and 8 . Patients were assessed at weeks 2, 4, 6, 8, 10, and 12. The IBDQ was administered to assess quality of life at weeks 0,6 , and 10 . The primary outcome variable was response (defined as a reduction of $>70$ points in the CDAI score from baseline) and remission at week 10 (defined as a CAI score of less than 150). Other secondary outcome variables included serum levels of CRP and mean lymphocyte counts. Remission occurred at week 10 in $37 \%$ of natalizumab-treated patients and $30 \%$ of patients receiving placebo $(p=0.12)$. A clinical response at week 10 was observed in $56 \%$ of patients treated with natalizumab and $49 \%$ of patients receiving placebo $(\mathrm{p}=0.05)$. However, a subgroup analysis of 660 patients including those with active inflammation at baseline (CRP concentration above the upper limit of normal) showed clinically and statistically significant differences in response and remission rates. Among patients with elevated CRP concentrations at baseline $58 \%$ of natalizumab treated patients and $45 \%$ of patients in the placebo group experienced a clinical response at week $10(\mathrm{p}<0.05)$, while $40 \%$ and $28 \%$ respectively, entered clinical remission $(\mathrm{p}<0.05)$.

Patients who reached the primary end point of response at week 10 in the ENACT-1 study were randomly reassigned in the ENACT-2 study to receive either natalizumab or placebo every 4 weeks through week 56 . Although the ENACT-1 trial did not meet the primary end point of response at week 10 with statistical significance in the overall population, the rates of sustained response during the ENACT-2 study were significantly higher among those who continued to receive natalizumab rather than being given placebo (61\% vs $28 \%$; $\mathrm{p}<0.001)$. The median time to the loss of response was also much longer among those receiving the antibody. The higher frequency of smokers among those receiving placebo in the ENACT-2 study, as compared with those receiving natalizumab (26\% vs 16\%), may have confounded the results, since smoking has been associated with increased CD activity.

\section{Study of Targan et al (2007)}

The ENCORE (Efficacy of Natalizumab in Crohn's disease Response and Remission) study was designed to confirm the hypothesis generated from the subgroup analysis of the ENACT-1 trial that natalizumab is effective as an induction therapy in patients with moderately to severely active CD and active inflammation characterized by elevated CRP concentrations. In the ENCORE study, 509 adult patients (from 832 patients screened) with moderately to severely active $\mathrm{CD}$ and objective evidence of active inflammation (elevated CRP) were recruited from 114 centers in North America, Europe, and selected countries around the world for a 12-week induction trial. Patients with a CDAI score between 220 and 450 and CRP levels greater than the upper limit of normal were randomized to receive an infusion of either $300 \mathrm{mg}$ natalizumab $(n=259)$ or placebo $(n=250)$ for a total of 3 infusions given at weeks 0,4 , and 8 . Efficacy and safety were assessed at weeks 4,8 , and 12 . The demography characteristics of all patients at baseline were: $50 \%(254 / 509)$ with ileocolonic location; 26.4\% (134/509) with colonic alone location; $24.7 \%$ (121/509) with ileal alone location. The primary outcome variable was clinical response by week 8 (defined as a reduction of $>70$ points in the CDAI score from baseline that was sustained through week 12). Secondary outcome variables included the proportion of patients achieving a clinical remission by week 8 and through week 12 and the proportion of patients in response or remission at any assessment, without considering mucosal healing as an important outcome.

The primary outcome (clinical response at week 8 sustained through week 12) occurred in 48\% (124 of 259) of natalizumab-treated patients compared with $32 \%$ (81 of 250 ) of patients receiving placebo $(\mathrm{p}<0.001)$. The secondary end point, remission at week 8 sustained through week 12 , occurred in $26 \%$ (68 of 259) of patients randomized to receive natalizumab and $16 \%(40 / 250)$ of patients who received placebo $(\mathrm{p}=0.002)$. At week 12 (secondary end point) $60 \%(155 / 259)$ of patients receiving natalizumab vs $44 \%$ $(109 / 250)$ of those administered placebo $(\mathrm{p}<0.001)$ were in response and 38\% (97/259) vs 25\% (63/250) respectively $(\mathrm{p}=0.001)$ were in remission. Treatment effect was evident as early as the first assessment. The median time to clinical response for patients in the natalizumab treatment group was 31 days compared with 51 days in the placebo group. The median time to clinical remission was 86 days for patients receiving natalizumab. At week 12 a greater improvement in mean total IBDQ score was observed among patients in the natalizumab group compared with those in the placebo group ( $\mathrm{p}<0.001)$. During the study, mean CRP concentrations consistently decreased from baseline values for patients who received natalizumab, while CRP concentrations remained 
essentially unchanged or slightly increased for those who received placebo. At week 12, 23\% of natalizumab-treated patients had CRP concentrations below the upper limit of normal compared with $8 \%$ of patients receiving placebo $(\mathrm{p}<0.001)$. Adverse events occurred at similar frequencies in the placebo and natalizumab groups, and no deaths occurred during this short-term induction study. Nine percent of patients in the natalizumab group and $13 \%$ of patients in the placebo group discontinued treatment due to an adverse events $(p=N S)$. Serious adverse events occurred in $5 \%$ of patients in the natalizumab group compared with $10 \%$ in the placebo group (such as exacerbation of $\mathrm{CD}$, one basal cell carcinoma, B-cell lymphoma in patient receiving a combination therapy with 6-mercaptopurine). Infections occurred in a greater proportion of patients in the natalizumab group than placebo group (35\% vs 30\%, respectively). This was primarily due to the more frequent occurrence of nasopharyngitis in the natalizumab group compared with placebo group. No opportunistic infections, including progressive multifocal leukoencephalopathy (PML), occurred during the study. Acute infusion reactions occurred in $9 \%$ of patients treated with natalizumab compared with $7 \%$ of the placebo group. Acute hypersensitivity-like reactions were determined before unblinding by a medical review of all adverse reactions that led to the interruption or discontinuation of study drug (adverse reactions that occurred within 24 hours of an infusion). This reaction occurred in $4 \%$ patients treated with natalizumab and $<1 \%$ in the placebo group. The positivity to anti-natalizumab antibodies was found in $9.5 \%$ patients. Concomitant immunosuppressive and corticosteroid therapy slightly decreased the rate of formation of anti-natalizumab antibodies. The presence of anti-natalizumab antibodies did not appear to have any impact on the overall incidence of adverse events, but seem to cause more acute infusion reactions and hypersensitivity-like reactions. The presence of antibodies did not appear to significantly affect response rates in this short-term study.

\section{Adverse events}

Natalizumab was generally well tolerated and the safety profile observed in the four included studies was similar. Adverse events occurred infrequently and were experienced by a similar proportion of natalizumab and placebo-treated patients. Presenting in more than $10 \%$ during the clinical trials were: headache, worsening of $\mathrm{CD}$, abdominal pain, artharlgia, colitis, influenza syndrome, infection, nausea, vomiting, fatigue, pharyngitis, infusion reactions, hypersensitivity-like reactions, and the development of antibodies against natalizumab (MacDonald and McDonald 2007). This suggests that the antibody did not interfere with neutrophil functions that are independent of $\alpha 4$ integrins and are necessary to combat bacterial and fungal infections. Concomitant use of immunosuppressive agents and corticosteroids may be protective against anti-natalizumab antibody formation, but in the ENACT trials the low overall rates of immunogenicity precluded statistical comparisons (Sandborn et al 2005). When persistent anti-natalizumab antibodies were present, they appeared to be associated with infusion reactions, hypersensitivity reactions, and loss of efficacy. All of the included trials lacked adequate power to detect rare or serious adverse events (Sandborn et al 2005). Chronic inhibition of integrins action could also have undesirable effects that are independent of the immunogenicity of the pharmacological inhibitor. In fact, integrins are required also for normal morphogenesis of the inner cell mass and are essential mediators of growth and survival of cells of the inner cell mass (Stephens et al 1995). This could suggest avoiding the use of agents that inhibit these pathways during pregnancy.

Some opportunistic infections seem to be selectively associated with individual treatments, presumably illuminating mechanisms important in specific immune defence; for example, the reactivation of latent tuberculosis after anti-TNF therapy, illuminating the key role played by $\mathrm{TNF} \alpha$ in the control of mycobacyteria and other intracellular pathogens (Podolsky 2005).

Recently, 2 patients with multiple sclerosis treated with natalizumab in combination with interferon $\beta$-1a and 1 patient with $\mathrm{CD}$ treated with natalizumab in combination with azathioprine developed progressive PML resulting in 2 patient deaths (Kleinschmidt-DeMasters and Tyler 2005; Langer-Gould et al 2005; Van Assche et al 2005). The CD patient received 3 doses of natalizumab in combination with azathioprine during ENACT-1, 9 doses of placebo in combination with azathioprine during ENACT-2, and 5 doses of natalizumab without concurrent azathioprine during an open label extension study (Sandborn et al 2005).

PML is a serious opportunistic infection of the central nervous system for which there is no specific treatment. PML is caused by the reactivation of quiescent JC polyomavirus. $\mathrm{JC}$ virus is a ubiquitous infection acquired during childhood that remains dormant in bone marrow, kidney epithelia, spleen, and gastrointestinal tract. Antibodies against JC virus are detectable in at least $80 \%$ of adults (Berger and Koralnik 2005). However, humoral immunity is insufficient to prevent the spread of the virus to the central nervous system. 
Activation of the latent virus and subsequent dissemination have been causally linked to the development of PML. Intermittent reactivation, with shedding of live virus in the urine, has been well documented in cross-sectional studies in healthy adults and pregnant women, but the phenomenon is poorly understood. Spread of the virus to the central nervous system and the subsequent development of PML occur in immunocompromised people, most commonly those infected with HIV, but also in some patients with lymphoma, sarcoidosis, and medication-induced immunosuppression (Berger and Koralnik 2005). In response to the problem the manufactures of natalizumab in consultation with regulatory authorities and the National Institutes of Health conducted a retrospective investigation to assess the risk of PML in natalizumab-treated patients (Yousry et al 2006). All adverse events were reviewed to identify any unrecognized occurrences of PML, and exposed patients underwent neurological examination and magnetic resonance scanning. A total of 3826 patients who had participated in recent clinical trials of natalizumab (multiple sclerosis, CD, and rheumatoid arthritis) were recruited for the evaluation. The incidence of PML associated with exposure to natalizumab is 1.0 cases per 1000 patients (95\% CI $0.2-2.8$ per 1000$)$ in this population who received a mean of 17.9 monthly doses of natalizumab. The investigation found no new confirmed cases of PML. The duration of exposure required to put patients at risk for PML is not known. Patients who were diagnosed with PML received 8-37 monthly infusions. The median exposure in the evaluated patients was 30 infusions among multiple sclerosis patients and 7 among CD or rheumatoid arthritis patients (Yousry et al 2006). Some authors (Adelman et al 2005; Berger and Koralnik 2005) suggest that the prospective measurement of the JC viral load in plasma and the reduction or discontinuation of natalizumab treatment, if JC virus is detected in the blood, might prevent the development of PML in this setting. A similar method has been used successfully to prevent nephropathy caused by the JC virus-related BK polyomavirus in kidney-transplant patients (Brennan et al 2005). Further analysis of serum samples obtained from the investigation will help to determine the feasibility of preventive strategies against the development of PML in patients treated with natalizumab or related drugs in the future. Finally, it should be noted that JC virus has also been potentially linked to gastrointestinal tract cancers, particularly those of the colon (Casini et al 2005; Niv et al 2005); reactivation of the virus may contribute to the chromosomal-instability pathway. If correct, this would raise at least a hypothetical concern about the use of this agent in patients with IBD.

\section{Critical comments and conclusions}

Pooled data from the four studies (MacDonald and McDonald 2007) suggest that natalizumab is effective for induction of clinical response and remission in some patients with moderately to severely active CD (remission [NNT $=17$ ] at 2 weeks and response at 4 weeks with 1 infusion $300 \mathrm{mg}$ or 3-4 mg/kg; remission [NNT $=10$ ] at 6 weeks with 2 infusions 3 or $4 \mathrm{mg} / \mathrm{kg}$; remission [NNT $=12$ ] and clinical response $[\mathrm{NNT}=10]$ at 12 weeks with 3 infusions) (these comparisons were not statistically significant at 10 weeks follow-up).

Natalizumab is particularly beneficial for patients with active inflammation or chronically active disease despite the use of conventional therapies (MacDonald and McDonald 2007). Indeed, subgroup analyses demonstrated significantly greater clinical response and remission rates for natalizumab compared with placebo in patients with elevated CRP levels, and active disease despite the use of immunosuppressant or prior anti-TNF $\alpha$ therapy (MacDonald and McDonald 2007). These benefits were apparent for both short-term and longterm treatment (clinical remission $[\mathrm{NNT}=8]$ and response $[\mathrm{NTT}=7]$ at 10 and 12 weeks with 3 infusions of natalizumab $4 \mathrm{mg} / \mathrm{kg}$ without previous different therapies; NNT $=6$ in patients using immunosuppressants and $\mathrm{NNT}=5$ in patients previously treated with anti-TNF $\alpha$ therapy). The ENCORE study supports the efficacy of natalizumab as induction therapy for $\mathrm{CD}$ patients with active inflammation. Indeed, 3 infusions of natalizumab (300 mg) were also significantly superior to placebo for induction of clinical remission and response at 8 and 12 weeks (Targan et al 2007) in patients with high CRP. Although natalizumab is finding a new role in the management of $\mathrm{CD}$, many problems about its utilization in $\mathrm{CD}$ remain unsolved and need to be discussed.

\section{Placebo response}

The use of placebo in randomized controlled trials is the gold standard for evaluating the effectiveness of new therapies. However, the placebo response rate is variable and may be affected by multiple factors. There were pronounced placebo remission and response rates in the natalizumab studies. In the Ghosh et al 2003 trial the remission and response rates increased in the placebo group from week 2 to week 12 (from $10 \%$ to $27 \%$ for remission rate and from $30 \%$ to $43 \%$ for response rate). In the ENACT-1 trial there was a high placebo response rate (49\%) compared to natalizumab (56\%) at 10 weeks, making it difficult to observe a treatment effect (Sandborn et al 2005). The high frequency and durability of the placebo response in the ENACT-2 study arouse concern that the results may have been partially confounded by the 
enrolment of some patients with little or no active disease. In the most recent trial (Targan et al 2007) the response rate at week 8 occurred in $32 \%$ of placebo group and this value was increasing at 12 weeks with $44 \%$ with clinical response and $25 \%$ with remission in the placebo group. Knowledge of the placebo outcomes and understanding specific study features that influence these outcomes is important for designing future clinical trials evaluating therapy.

\section{Dosage and timing schedule}

The optimal dosage and timing schedule for induction of remission and response using natalizumab therapy remains to be completely determined. Ghosh et al (2003) looked at the efficacy of 2 different dosages of natalizumab in comparison to placebo and did not find any advantage for 2 infusions of $6 \mathrm{mg} / \mathrm{kg}$ natalizumab over $3 \mathrm{mg} / \mathrm{kg}$. Three of the included studies dosed patients every 4 weeks for a total of 2 infusions and 3 infusions. No studies to date have tried a different timing schedule.

\section{Disease behavior and location}

The ENACT-1/2 and ENCORE trials (Sandborn et al 2005) did not enrol patients with fistulazing CD. Although Gordon et al (2001) and Ghosh et al (2003) included patients with fistulizing $\mathrm{CD}$ (30\% and $15.3 \%$, respectively), they did not make any comments on the efficacy of natalizumab for fistula healing and closure. Moreover, there are no published data that consider the natalizumab efficacy on the basis of different patients behavior and location and their evolution during the disease history.

\section{Definition of outcome}

In all published studies mucosal healing was not evaluated as an outcome. Clinical remission and response were based on CDAI values and laboratories analyses. Considering the heterogeneity of CD, the assessment of remission is more complex. Since the CDAI relies heavily on symptomatic parameters, it does not come as a surprise that its correlation with endoscopic scores or histological activity is weak (Modigliani et al 1990; Oldenburg and Hommes 2007). Presently, an unambiguous clinical and endoscopic definition of remission in $\mathrm{CD}$ is lacking, hampering the efforts of clinicians to formulate clear therapeutic objective for the long term, especially in the evaluation of new biological therapies efficacy in clinical trials.

\section{Previous therapy}

In all published studies the median time of wash-out of immunosuppressive therapy was within 2 or 4 months from the starting time of stable dosage (from $38 \%$ to $17 \%$ in the placebo group and from $38 \%$ to $18 \%$ in the natalizumabtreated group of patients with immunosuppression therapy). In the ENCORE study there was no specification about the time needed to consider a stable dosage of previously started immunosuppressive therapy. Considering the inclusion criteria there was a short time to wash-out with the previous treatment (steroids and immunosuppressive drugs) which can influence the results about the efficacy of natalizumab. It is important to highlight that the effect of the immunosuppressive drugs starts after 2-6 months of treatment. This could be too short a time to be sure about the independent effect of natalizumab.

\section{Adverse events}

Natalizumab was approved by the FDA for the treatment of relapsing-remitting multiple sclerosis in December 2004. Its use in clinical practice and clinical trials was suspended in February 2005 because of the development of PML in patients treated with natalizumab (MacDonald and McDonald 2007). The FDA approved natalizumab for CD in January 2008, and approved a supplemental biologics licence application for the reintroduction of natalizumab monotherapy treatment for relapsing-remitting multiple sclerosis in June 2006. Natalizumab was also approved for this indication by the European Medicines Evaluation Agency (EMEA) in June 2006 and Health Canada in October 2006 (MacDonald and McDonald 2007). Actually we cannot neglect the previous reported observation of PML cases after natalizumab treatment. These observations provide a unique and unexpected window into our understanding of the pathogenesis of PML and force us to reconsider the potential risks associated with the inhibition of lymphocyte trafficking. Our understanding of the ratio between benefits and risks of natalizumab in patients with CD is not sufficient to offer a definitive answer. Efforts should be focused not only on defining the true therapeutic efficacy of this but also on gaining a more precise understanding of the mechanisms of action and risk of treatment with antibody against $\alpha 4$ integrins. For these reasons, natalizumab should be targeted as a second-line biological therapy to patients who have frequent and debilitating recurrences of CD with close surveillance by magnetic resonance scanning and serum analysis for the earliest signs of JC virus (Podolsky 2005). In conclusion, it is important in the future to stay mindful of defining the true therapeutic efficacy and on gaining more precise understanding of the mechanisms of action and risks of treatment with antibody against $\alpha 4$-integrin and with other new biological agents developed. 


\section{Abbreviations}

CD, Crohn's disease; CDAI, Crohn's Disease Activity Index; CRP, C-reactive protein; EMEA, European Medicines Evaluation Agency; ENCORE, Efficacy of Natalizumab in Crohn's disease Response and Remission; FDA, Food and Drug Administration; IBD, Inflammatory bowel disease; IBDQ, Inflammatory Bowel Diseases Questionnaire; MadCAM-1, Mucosal addressin-cell adhesion molecule-1; NS, Non significant p value; PML, Progressive multifocal leukoencephalopathy; TNF, Tumour necrosis factor; UC, Ulcerative colitis; VCAM-1, Vascular-cell adhesion molecule-1.

\section{References}

Adelman B, Sandrock A, Panzara MA. 2005. Natalizumab and progressive multifocal leukoencephalopathy. $N$ Engl J Med, 353:432-3.

Alon R, Kassner PD, Carr MW, et al. 1995. The integrins VLA-4 supports tethering and rolling in flow on VCAM-1. J Cell Biol, 128: 1243-53.

Berger JR, Koralnik IJ. 2005. Progressive multifocal leukoencephalopathy and natalizumab-unforeseen consequences. $N$ Engl $J$ Med, 353:414-6.

Berlin C, Bargatze RF, Campbell JJ, et al. 1995. Alpha4 integrins mediate lymphocyte attachment and rolling under physiologic flow. Cell, 80:413-22.

Binion DG, West GA, Volk EE, et al. 1998. Acquired increase in luokocyte binding by intestinal microvascular endothelium in inflammatory bowel disease. Lancet, 352:1742-6.

Brennan DC, Agha I, Bohl DL, et al. 2005. Incidence of BK with tracolimus versus cyclosporine and impact of preemptive immunosuppression reduction. Am J Transplant, 5:582-94.

Butcher EC, Picker LJ. 1996. Lymphocyte homing and homeostasis. Science, 272:60-9.

Casini B, Borghese L, Del Nonno F, et al. 2005. Presence and incidence of DNA sequences of human polyomavirus $\mathrm{BKV}$ and JCV in colorectal tumor tissue. Anticancer Res, 25:1079-85.

Elan Pharma (Europe) Ltd. 1997. A phase I double-blind, placebo-controlled, ascending single intravenous dose, safety, tolerability, pharmacokinetic, immunogenicity and potency study in healthy male volunteers. Athena report nr. AN100226-101.

Fiocchi C. 1998. Inflammatory bowel disease:etiology and pathogenesis. Gastroenterology, 115:182-205.

Gosh S, Goldin E, Gordon FH, et al. 2003. Natalizumab for active Crohn's disease. $N$ Eng J Med, 348:24-32.

Gordon FH, Clement WYL, Hamilton MI, et al. 2001. A randomized placebo-controlled trial of a humanized monoclonal antibody to alpha4 integrin in active Crohn's disease. Gastroenterology, 121:268-74.

Gordon FH, Hamilton MI, Donoghue S, et al. 2002. A pilot study of treatment of active ulcerative colitis with natalizumab, a humanized monoclonal antibody to alpha4 integrin. Aliment Pharmacol Ther, 16:699-705.

Hesterberg PE, Winsor-Hines D, Briskin MJ, et al. 1996. Rapid resolution of chroic colitis in the cotton-top tamarin with an antibody to a gut-homing integrin alpha 4 beta 7. Gastroenterology, 111:1373-80.

Hynes RO. 2002. Integrins:bidirectional, allosteric signalling machines. Cell, 110:673-87.
Kleinschmidt-DeMasters BK, Tyler KL. 2005. Progressive multifocal leukoencephalopathy complicating treatment with natalizumab and interferon beta-1a for multiple sclerosis. $N$ Engl J Med, 353:369-74.

Kubes P, Niu XF, Smith CW, et al. 1995. A novel beta 1-dependent adhesion pathway on neutrophils:a mechanism invoked by dihydrocytochalasin $\mathrm{B}$ or endothelial transmigration. FASEB J, 9:1103-11.

Langer-Gould A, Atlas SW, Green AJ, et al. 2005. Progressive multifocal leukoencephalopathy in a patient treated with natalizumab. $N$ Engl $J$ Med, 353:375-81

MacDonald JK, McDonald JWD. 2007. Natalizumab for induction of remission in Crohn's disease. Cochrane Database of Systematic Reviews, 2007 Issue 1.

Miller DH, Khan OA, Sherenta WA, et al. 2003. A controlled trial of natalizumab for relapsing multiple sclerosis. $N$ Engl J Med, 348:15-23.

Modigliani R, Mary JY, Simon JF, et al. 1990. Clinical biological and endoscopical picture of attacks of Crohn's disease:evolution on prednisolone. Groupe d'etude therapeutique des affections inflammatoires digestives. Gastroenterology, 98:811-8.

Nakamura K, Honda K, Mizutani T, et al. 2006. Novel strategies for the treatment of inflammatory bowel disease:selective inhibition of cytokines and adhesion molecules. World J Gastroenterol, 12:4628-35.

Niv Y, Goel A, Boland CR. 2005. JC virus and colorectal cancer :a possible trigger in the chromosomal instability pathways. Curr Opin Gastroenterol, 21:85-9.

Oldenburg B, Hommes D. 2007. Biological therapies in inflammatory bowel disease:top-down or bottom-up? Curr Opin Gastroenterol, 23:395-99.

Picarella D, Hurbut P, Rottman J, et al. 1997. Monoclonal antibodies specific for beta 7 integrin and mucosal addressin cell adhesion molecule-1 (MAdCAM-1) reduce inflammation in the colon of scid mice reconstituted with CD45RBhighCD4+ T-cells. J immunol, 158:2099-106.

Podolsky DK, Lobb R, King N, et al. 1993. Attenuation of colitis in the cotton-top tamarin by anti-alpha 4 integrin monoclonal antibosy. $J$ Clin Invest, 92:372-80.

Podolsky DK. 2005. Selective adhesion-molecule therapy and inflammatory bowel disease-a tale of Janus? N Engl J Med, 353:1965-8.

Sandborn WJ, Colombel JF, Enns R, et al. 2005. Natalizumab induction and maintenance therapy for Crohn's disease. $N$ Engl J Med, 353:1912-25.

Soriano A, Salas A, Salas A, et al. 2000. VCAM-1, but not ICAM-1 or MAdCAM-1, immunoblockade ameliorates DSS-induced colitis in mice. Lab Invest, 80:1541-51.

Springer TA. 1994. Traffic signals for lymphocyte recirculation and leukocyte emigration:the multistep system. Cell, 76:301-14.

Stephens LE, Sutherland AE, Klimanskaya IV, et al. 1995. Deletion of beta 1 integrins in mice results in inner cell mass failure and peri-implantation lethality. Genes Dev, 9:1883-95.

Targan SR, Feagan BG, Fedorak RN et al. 2007. Ntalizumab for the treatment of active Crohn's disease:results of the ENCORE trial. Gastroenterology, 132:1672-83.

Van Assche G, Van Ranst M, Sciot R, et al. 2005. Progressive multifocal leukoencephalopathy after natalizumab therapy for Crohn's disease. N Engl J Med, 353:362-8.

Von Andrian UH, Mackay CR. 2000. T-cell function and migration:two sides of the same coin. N Engl J Med, 343:1020-34.

Yednock TA, Cannon C, Frits LC, et al. 1992. Prevention of experimental autoimmune encephalomyelitis by antibodies against alpha4beta 1 integrin. Nature, 356:63-6.

Yousry TA, Habil M, Majot EO, et al. 2006. Evaluation of patients treated with natalizumab for progressive multifocal leukoencephalopathy. N Engl J Med, 354:924-33. 